\title{
GLAD!
}

Revue sur le langage, le genre, les sexualités

$04 \mid 2018$

Rhétoriques antiféministes

\section{Rhétoriques antiféministes : entre recherche et pratiques}

Antifeminist Rhetorics: Research and Practice

Auréline Cardoso et Charlotte Thevenet

\section{OpenEdition}

1 Journals

Édition électronique

URL : http://journals.openedition.org/glad/995

DOI : $10.4000 /$ glad.995

ISSN : 2551-0819

Éditeur

Association GSL

Référence électronique

Auréline Cardoso et Charlotte Thevenet, «Rhétoriques antiféministes : entre recherche et pratiques », GLAD! [En ligne], 04 | 2018, mis en ligne le 30 juin 2018, consulté le 17 décembre 2020. URL : http:// journals.openedition.org/glad/995; DOI : https://doi.org/10.4000/glad.995

Ce document a été généré automatiquement le 17 décembre 2020.

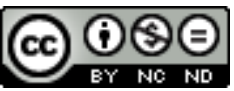

La revue GLAD! est mise à disposition selon les termes de la Licence Creative Commons Attribution Pas d'Utilisation Commerciale - Pas de Modification 4.0 International. 


\section{Rhétoriques antiféministes : entre recherche et pratiques}

Antifeminist Rhetorics: Research and Practice

Auréline Cardoso et Charlotte Thevenet

Femme sans teste, tout en est bon. (Proverbe misogyne) 
Femme sans teste tout en est bon [estampe]

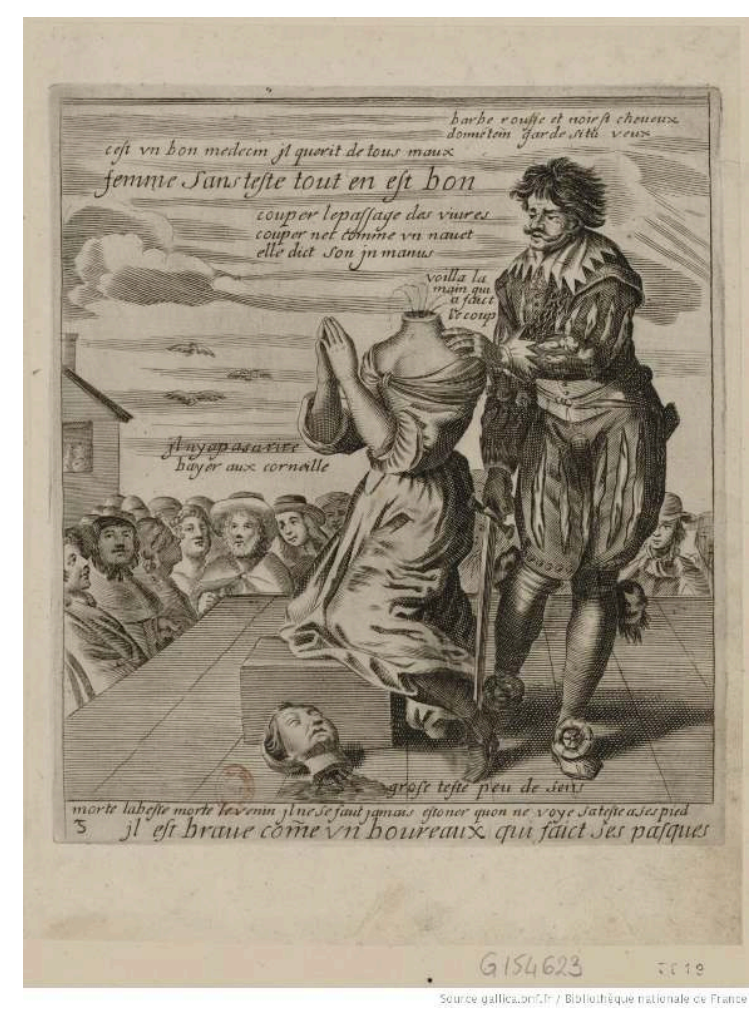

Recueil des plus illustres proverbes divisés en trois livres. Le second livre ou est despaint les proverbes joyeux (impr. à Paris par I. Lagnet, 1657)

Crédits : Gallica (BNF)

1 En mai 2016, à l'Université de Toulouse II Jean Jaurès, se tenaient deux journées d'études intitulées "Rhétoriques antiféministes: entre recherche et pratiques", organisées par le collectif de jeunes chercheuses féministes Arpège-EFiGiES Toulouse ${ }^{1}$, et qui mettaient en dialogue les savoirs issus d'universitaires, de militantes et/ou de professionnelles d'organisations féministes. Deux ans plus tard, alors que les médias saluent d'une main la « libération de la parole » symbolisée par le hashtag \#MeToo, et publient de l'autre articles et pièces d'opinion qui condamnent cette supposée libération, il semble plus urgent que jamais de mettre les discours antiféministes sur la table de dissection critique. Non seulement l'actualité de ces derniers mois a-t-elle rappelé, s'il en était besoin, combien les discours réactionnaires et antiféministes étaient prompts à la riposte, mais également combien ils étaient nombreux et divers : venus d'hommes de droite comme de gauche, bien sûr, mais aussi de femmes comme l'a montré en fanfare la tribune réclamant « la liberté d'importuner » parue dans Le Monde en janvier, voire, pourquoi pas, de femmes se réclamant du féminisme ${ }^{2}$. Face au foisonnement et au succès de ces discours aujourd'hui, face aussi à leur constance dans l'histoire, ce numéro thématique fruit de la collaboration des membres du collectif Arpège-EFiGiES Toulouse et de l'équipe éditoriale de GLAD!, vise à offrir une anatomie critique des discours antiféministes aujourd'hui et dans l'histoire, qui rende compte de leur permanence autant que de la diversité de leurs rhétoriques.

2 Il s'agit en effet de penser ensemble les diverses réalités que recouvrent les antiféminismes aujourd'hui, «nébuleuse aux manifestations tangibles » (Devreux \& Lamoureux 2012) certes, mais dont les contours restent néanmoins flous. Des pères 
divorcés aux magazines féminins, en passant par le milieu universitaire aujourd'hui, les contributions illustrent toute une palette de sphères d'actuation des discours antiféministes. La perspective historique adoptée dans certains articles permet quant à elle de saisir les (dis)continuités de ces rhétoriques, et les perpétuels renouvellements de discours dont le fond et la forme varient, mais pas l'objectif, puisqu'il s'agit, in fine, de s'opposer aux avancées sociales en faveur de l'égalité de genre et de perpétuer la domination d'un genre sur l'autre. La richesse de ces matériaux empiriques permet une compréhension fine de la reproduction des discours antiféministes, et des manières dont ils s'entrecroisent avec d'autres rhétoriques réactionnaires (Hirschman 1991), telles que les rhétoriques racistes, transphobes et néo-libérales. Ce dossier entend donc participer à la construction des savoirs sur les antiféminismes, qui, s'ils font l'objet d'un nombre croissant de recherches en France ${ }^{3}$, ne cessent de se renouveler et demandent ainsi une attention et une vigilance féministes accrues.

\section{Une multitude d'antiféminismes}

3 La manière de définir l'antiféminisme varie selon l'angle sous lequel il est appréhendé. Ainsi, en écrivant que l'antiféminisme est une réaction d'hostilité et de rejet à l'égard du féminisme, Christine Bard met l'accent sur le caractère défensif de l'antiféminisme et sur la dynamique relationnelle entre mouvement féministe et contre-mouvement antiféministe (Bard 1999). D'autres conceptualisations plus larges de l'antiféminisme mettent en exergue sa dimension " ordinaire ", en définissant l'antiféminisme comme « une expression directe de la misogynie » et « l'argumentaire politique de la haine des femmes " (Dworkin 2012: 195), ou comme « un continuum allant de l'indifférence à la violence en passant par la non-reconnaissance des femmes comme égales, le mépris et l'hostilité déclarée » (Descarries 2015 : 75). Pour Diane Lamoureux et Francis DupuisDéri, si l'antiféminisme peut avoir des effets pour l'ensemble des femmes, il relève toutefois d'une logique distincte de la misogynie et vise prioritairement les féministes (Lamoureux \& Dupuis-Déri 2015).

4 Tout comme le mouvement féministe, la nébuleuse antiféministe (Devreux \& Lamoureux 2012) présente une grande pluralité d'objectifs, de répertoires d'actions ou de principes idéologiques. On peut tout d'abord distinguer l'antiféminisme ordinaire, renvoyant aux discours de sens commun qui s'opposent, plus ou moins frontalement, aux revendications féministes; ensuite, l'antiféminisme religieux ou conservateur et s'attaquant plus directement aux droits sexuels et reproductifs et visant les féministes ; enfin, le masculinisme, qui au moyen d'actions plus ou moins virulentes revendique de «nouveaux droits» pour les hommes et notamment les pères (Blais 2012). On peut également mentionner l'antiféminisme d'État ou plutôt l'antiféminisme dans l'État, qui désigne et permet d'exposer la façon dont des agents de l'État ou acteurs et actrices politiques, font barrage aux revendications féministes ou au travail d'associations et institutions œuvrant pour l'égalité (Dupuis-Déri 2013).

5 En France, l'antiféminisme est représenté d'abord par des groupes comme SOS Papa ou le groupe anti-IVG Les Survivants, qui, bien que minoritaires, parviennent à occuper un espace médiatique certain. Mais les idées antiféministes imprègnent en fait les discours de nombreux et nombreuses intellectuel-le-s, politiciennes et politiciens (Andriamandroso 2017 ; Dauphin 2010) ; l'antiféminisme fonctionne comme un vecteur d'union sacrée de tout le spectre politique, comme l'anti-suffragisme mettait d'accord 
l'Action française et le Parti Radical au début du $\mathrm{xx}^{\mathrm{e}}$ siècle ${ }^{4}$. Certaines formes d'antiféminisme peuvent également influencer les pratiques professionnelles, par exemple dans le champ du travail social lorsque la lecture féministe des violences conjugales est évacuée au profit d'une approche psychologisante et inter-individuelle qui minimise les rapports de pouvoir liés au genre (Casas Vila 2006; Côté \& Lapierre 2017). Pour autant la plupart de ces personnes « relais » d'idées hostiles aux féminismes ne se pensent pas nécessairement comme antiféministes, et c'est peut-être dans ce paradoxe que réside toute la difficulté d'étudier l'antiféminisme : il est à la fois la toile de fond d'une multitude de discours et de pratiques, tout en étant dilué dans d'autres logiques notamment néo-libérales (Blais 2017) et en avançant masqué derrière des valeurs égalitaires (Lefort 2017). Le paradoxe est poussé à son comble quand c'est au nom du féminisme que s'articule une rhétorique antiféministe, comme c'est le cas dans ce qu'on peut appeler les nouveaux (anti)féminismes de droite, représentés par le " féminisme intégral » de Marianne Durano et de la revue Limite. Il y a quelques années, un mouvement comme celui des "Antigones", groupe de jeunes femmes toujours habillées d'un blanc virginal et prônant " la féminité pour les femmes ", posait déjà la question des rhétoriques antiféministes venues des femmes au nom de valeurs supposément « féminines ».

\section{Déconstruire les discours antiféministes}

6 Si ce dossier propose de se pencher sur les discours antiféministes, c'est notamment parce que c'est bien par le langage, les discours (écrits ou oraux, médiatiques, scientifiques, religieux...) que se diffuse l'antiféminisme. La palette argumentative est large, allant d'une dénonciation des supposés privilèges féminins à la valorisation de la " complémentarité entre les femmes et les hommes », en passant par la revendication de l'égalité parentale. Pour autant, malgré cette diversité de stratégies, on peut repérer un certain nombre de récurrences dans les rhétoriques antiféministes.

7 Francine Descarries distingue trois registres argumentatifs de l'antiféminisme ordinaire : la distorsion (distorsion des faits comme lorsqu'il est affirmé que l'égalité est atteinte), la simplification abusive (lorsque l'égalité est considérée comme une affaire privée et que le féminisme est vidé de son contenu politique et transformateur), et la victimisation (lorsque l'Histoire est réinterprétée pour faire des hommes les principales victimes des changements sociaux récents) (Descarries 2005). Les interprétations fallacieuses de statistiques, voire leur négation ou leur lecture sommaire, contribuent également à légitimer l'idée selon laquelle les luttes féministes auraient été menées au-delà du nécessaire et auraient abouti à l'avènement d'un «matriarcat » où les hommes n'auraient plus leur place (Bard 1999; Blais \& DupuisDéri 2012). On peut également mentionner le recours fréquent à la mise en exergue de l'exemple marginal d'une femme ayant accédé à un poste de pouvoir (une femme présidente, cheffe d'entreprise) pour " prouver » qu'il y aurait bien une féminisation de la société et que les femmes voire les féministes seraient désormais aux commandes (Blais 2012 ; Rabenoro 2012). Quant aux discours plus spécifiquement masculinistes, en plus de la distorsion et de la victimisation, ils mobilisent souvent le témoignage individuel d'un homme en souffrance construit comme une « victime impuissante » qui serait représentatif d'une condition masculine en déclin, comme le montre Étienne Lefort dans son article « Construire les hommes comme des victimes irresponsables. Les 
stratégies discursives des associations masculinistes françaises ». Les thèmes privilégiés par les rhétoriques masculinistes, largement diffusés par les magazines féminins (Cardoso 2018 dans ce numéro), s'articulent autour de la «crise de la masculinité », de l'inégalité face à la justice des familles qui favoriserait les femmes, du suicide des hommes et de l'échec scolaire des garçons (Lamoureux 2008).

D'autres discours, sans être explicitement antiféministes, viennent délégitimer les revendications féministes et surtout le recours à l'action collective. C'est le cas des rhétoriques sur le "post-féminisme", notion aux contours flous qui est souvent associée à la proclamation de la mort du féminisme (Cossy et al. 2009; Aronson 2015) et au rejet de celui-ci par les jeunes femmes (Braithwaite 2004), et dont l'article de Mary Hawkesworth "Sémiotique de l'enterrement prématuré: le féminisme à l'ère du postféminisme " traduit dans ce numéro par Aude Ferrachat fait la généalogie et la critique. Le post-féminisme, topos des antiféminismes depuis les années 1970 comme le montre Audrey Lasserre dans son article «Des féministes qui ne sont pas féministes? Écrivaines et lutte des femmes dans la France des années 1970 », est considéré comme une nouvelle phase du mouvement des femmes, qui s'inscrirait dans une vision néolibérale des inégalités et proposerait de se concentrer sur des solutions individuelles (Aronson 2015 ; Genz 2006). En cela les rhétoriques post-féministes se rapprochent du "féminisme élitiste ", dont les discours et propositions, sur l'accès des femmes aux postes de pouvoir, concernent surtout la fraction diplômée et aisée des femmes (Klaus 2010).

9 La valorisation d'un mouvement "post-féministe » a également souvent pour effet de délégitimer le féminisme dit de la « deuxième vague », dont la radicalité et l'agressivité - largement exagérées - sont décriées, et dont la richesse politique et tactique est réduite à la visibilité des manifestations de rue (Hawkesworth 2004 traduit dans ce numéro). Là encore, cette stratégie s'inscrit dans une tradition au long cours de discours qui transforment volontiers les féministes en monstres, qu'il s'agisse de furies mettant des soutiens-gorge au bucher (Bard 2012), des «tricoteuses» de la Révolution si bien inscrites dans la mémoire misogyne par Dickens (A Tale of Two Cities), ou des "sorcières androphobes " qui persistent jusqu'au $\mathrm{xx}^{\mathrm{e}}$ siècle ${ }^{5}$. Les discours sur le postféminisme alimentent stratégiquement l'idée d'une rupture générationnelle, faisant des « jeunes femmes d'aujourd'hui » un bloc homogène qui ne se reconnaitrait pas dans le féminisme, sans définir ce qu'est le féminisme ni s'intéresser à ce qu'en perçoivent ces femmes nées après les mouvements politiques de libération des femmes (Aronson 2015 ; Hall \& Rodriguez 2003). Ainsi le post-féminisme serait une sorte de «nouveau » féminisme, plus joyeux, indépendant et non politique qui valoriserait la réussite individuelle et la compétition entre individu-e-s perçu-e-s comme également doté-e-s en opportunités (Blais \& Dupuis-Déri 2014 ; Braithwaite 2004 ; Lamoureux 2008), plutôt que la solidarité et la lutte collective (Dupuis-Déri 2015).

\section{Critique des discours antiféministes}

\section{L'argument de la nature}

10 À la lecture des différents articles, la récurrence de certains arguments et notamment d'une forme de «naturalisme différentialiste » se dégage d'emblée, comme l'explique Juliette Rennes dans l'entretien "Retour sur un siècle et demi de rhétorique anti- 
égalitaire et antiféministe ». Il s'agit là toujours selon Juliette Rennes d'une «matrice idéologique commune à tous les discours qui s'opposent aux réclamations féministes ", construisant les femmes et les hommes comme incommensurablement différents et dès lors, voué-e-s à occuper des fonctions distinctes, au sein de la famille comme du couple et du travail. "La différence est la façon dont [...] on justifie l'inégalité entre les groupes [...]» écrit Christine Delphy dans «Penser le genre» (2001: 8). Particulièrement paradigmatiques de cette stratégie, le mouvement de la « Manif Pour Tous » et plus largement l'offensive vaticane contre la dite "théorie du genre » sont auscultées dans ce numéro à travers la recension par Julie Abbou de l'ouvrage de Sara Garbagnoli et Massimo Prearo, La croisade "anti-genre" paru en 2017. On pourrait ajouter que la pensée de la différence vient justifier les mesures renforçant et/ou légitimant les politiques inégalitaires, comme lorsque les militants des groupes de pères séparés, étudiés par Aurélie Fillod-Chabaud dans l'article "L'antiféminisme d'État. Une analyse rhétorique du mouvement des pères séparés au Québec", revendiquent le retour à une éducation non mixte afin de mieux respecter les différences naturelles entre hommes et femmes. De façon autrement plus masquée, certains articles de magazines féminins s'emploient également à justifier des inégalités sociales (comme les inégalités liées au partage des tâches ménagères) en invoquant une différence ontologique et indépassable entre hommes et femmes; c'est ce que montre dans ce numéro Auréline Cardoso dans un article intitulé «Antiféminisme sur papier glacé. La rhétorique réactionnaire des magazines féminins». Contrepartie de cette naturalisation du genre, les femmes qui refusent d'y souscrire (qu'il s'agisse de féministes révolutionnaires ou de femmes philosophes comme chez Arnaud \& Gratton dans ce numéro) sont construites comme des aberrations de la nature ; c'est le stigmate que représente le mot même de "féministe" à travers l'histoire que retrace Caroline Fayolle dans son article « Des corps "monstres". Historique du stigmate féministe ».

\section{Pauvres hommes...}

Bon nombre d'articles explorent la stratégie d'inversion des rôles, opérée plus particulièrement par les masculinistes, représentés dans ce dossier au travers des militants des organisations de défense des droits des pères (articles d'Aurélie FillodChabaud et d'Étienne Lefort). Si ces groupes sont, pour l'heure, beaucoup moins audibles en France qu'outre-Atlantique, ils constituent cependant « la composante la plus active du mouvement masculiniste en France » (Lefort dans ce numéro). Au moyen notamment d'un usage fallacieux de statistiques, les membres de ces groupes cherchent à faire de ce qui est présenté comme un inexorable déclin de la condition masculine, un problème public d'ampleur que l'on ne saurait négliger. Les discours sur la résidence alternée qui serait presque systématiquement refusée, sur les violences conjugales qui toucheraient autant, sinon plus, les hommes que les femmes, ou sur les suicides masculins, participent d'une «stratégie victimaire » (Fillod-Chabaud dans ce numéro).

Le supposé acharnement des féministes à l'égard des hommes est lui aussi multiséculaire : dès la fin du $\mathrm{XIX}^{\mathrm{e}}$ siècle, le combat féministe est présenté comme une lutte " anti-hommes " par ses détracteurs, alors même que des hommes le soutenaient déjà (et le soutiennent encore), comme nous le rappelle Juliette Rennes. 


\section{Maquiller les discours antiféministes sous une apparence égalitaire ou progressiste}

Si l'on a parfois tant de peine à identifier les discours antiféministes, c'est aussi parce que leurs auteurs, bien conscients de la (relative) diffusion d'une norme égalitaire, prennent soin de ne pas s'opposer ouvertement aux droits des femmes et à l'égalité. Dès le Moyen-Âge tardif et la Renaissance, les auteurs antiféminins prenant position dans la Querelle des femmes sont ainsi contraints d'inclure dans leurs argumentaires polémiques certains des arguments qu'ils combattent; dans son article intitulé "Modalités de diffusion et rhétoriques des discours misogynes et misogames imprimés à la Renaissance ", Tatiana Clavier montre ainsi la circulation voire la réversibilité des discours misogynes à cette époque. Aussi ne s'agit-il pas forcément pour les antiféministes de nier la légitimité de la lutte pour l'égalité, mais d'affirmer que celle-ci est déjà advenue : Julienne Rennes rappelle la prégnance de la rhétorique de «l'égalité déjà là » identifiée par Christine Delphy (Delphy 2010) au xIx siècle, alors même que contrairement à aujourd'hui l'égalité femmes-hommes était loin d'être inscrite dans la loi. À cette contre-vérité s'en articule une autre : les discours proclamant « la mort du féminisme » (Hawkesworth dans ce numéro, traduit par Aude Ferrachat), dès les années soixante-dix, sont portés par des femmes, parfois même identifiées par d'autres comme féministes (c'est le cas d'Hélène Cixous), qui s'efforcent pourtant de rendre le terme caduc (Lasserre dans ce numéro). Que ce soit au nom d'une égalité débarrassée des rapports de domination telle qu'analysée par Hawkesworth, ou d'une radicalité plus radicale que la révolution comme chez certaines antiféministes des années 1970 (Lasserre dans ce numéro), le féminisme semble destiné à être stratégiquement conjugué au passé, renvoyé à Mathusalem et ainsi commodément privé de voix à la table du progrès.

\section{L'antiféminisme au croisement des rapports sociaux}

14 Le retentissement et les suites de ce qui a vite été qualifié d' "affaire Weinstein ${ }^{6}$ " viennent illustrer à quel point différents rapports sociaux, et notamment de classe et de race, s'imbriquent perpétuellement aux rapports sociaux de sexe et se renforcent mutuellement. Comme l'ont souligné plusieurs commentatrices ${ }^{7}$, si les dénonciations légitimes - de violences sexistes et sexuelles commises par un homme à l'encontre de dizaines de femmes ont pu trouver un tel écho et une telle écoute, c'est notamment parce que les victimes sont incarnées par des femmes riches, célèbres et majoritairement blanches. Se remémorer la réception des accusations de Nafissatou Diallo à l'encontre de Dominique Strauss-Khan suffit à se convaincre qu'en matière de dénonciation des violences, toutes les paroles sont loin de se valoir. De même, si ces différentes affaires permettent de pointer du doigt des auteurs issus de milieux favorisés, les politiques cherchant à répondre à la colère - elle aussi, toujours légitime - suscitée par ces révélations, en se focalisant sur ce que l'on nomme désormais le " harcèlement de rue ", ciblent toujours les mêmes coupables : des hommes plutôt issus des classes populaires et racisés, comme le souligne la tribune «Contre la pénalisation du harcèlement de rue » signée par des militantes et chercheuses engagées dans la lutte contre les violences envers les femmes. ${ }^{8}$. Dans cette perspective, certains textes du numéro fournissent réflexion et outils pour penser ces articulations et surtout pour élaborer des stratégies de défense tenant compte de l'imbrication des rapports sociaux. 
D'abord, l'analyse fournie dans l'article « Le traitement médiatique des violences faites aux femmes: entre instrumentalisation et invisibilisation" par les membres de l'association Faire Face, qui propose des stages d'auto-défense féministes, est salutaire en ce qu'elle permet de réfléchir à des stratégies féministes pour dénoncer et combattre les violences faites aux femmes, sans pour autant alimenter d'autres formes de violences (de classe et raciste en particulier). Comme en écho à ces réflexions, la recension par Mickaëlle Provost du livre d'Elsa Dorlin paru en 2017, Se défendre, propose de concevoir l'auto-défense des personnes minorisées à travers une lentille phénoménologique (Provost dans ce numéro). Dans un tout autre espace, celui de l'université, Sarah Arnaud et Cloé Gratton nous offrent un guide de survie pour femmes philosophes en milieu misogyne et antiféministe, dans un article intitulé «Les femmes en philo, qu'est-ce que ça mange en hiver? ». Enfin, le compte-rendu par Agathe Senna de la première édition en français d'essais rédigés en 1907 par He-Yin Zhen, féministe et anarchiste chinoise (rassemblés sous le titre La Revanche des femmes et autres textes aux éditions de l'Asymétrie en 2018, traduction de Pascale Vacher), propose un contrepoint radical et bienvenu à tous ces discours antiféministes, sous la forme d'une pensée décisivement émancipatrice et révolutionnaire.

\section{Opérateur Céphalique}

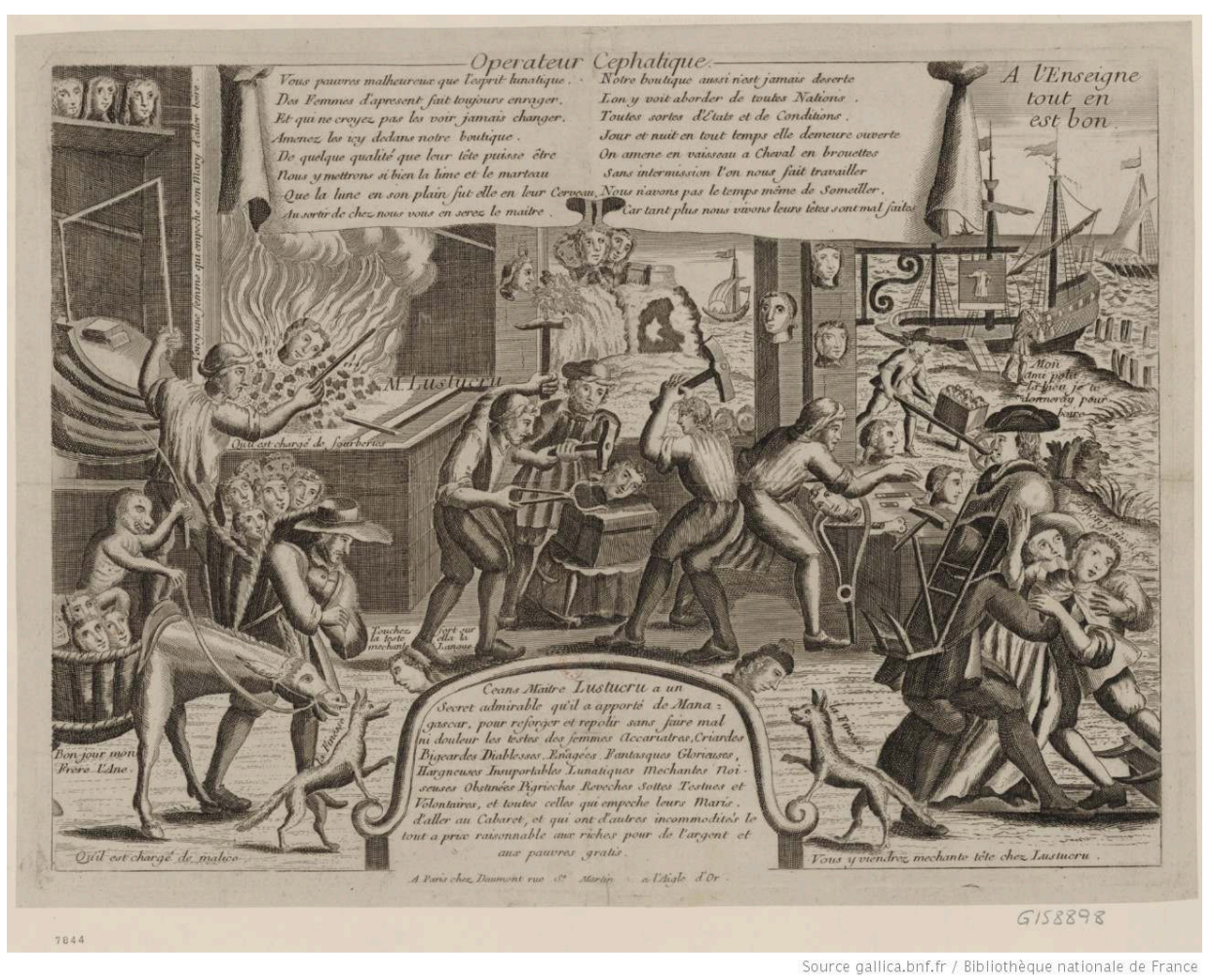

Opérateur Céphalique Céans maitre Lustucru a un secret admirable qu'il a apporté de Managascar pour reforger et repolir sans faire mal ni douleur les testes des femmes acariatres, criardes... [estampe] (publication à Paris chez Daumont, 1720)

Crédits : Gallica (BNF)

En plus de son dossier thématique, ce numéro \#4 poursuit le travail entrepris dans les numéros précédents. Travail d'exploration de formats de recherche inédits, d'abord: ainsi de la recherche filmée et montée d'Anne-Laure Vernet et de Luc Schicharin («Une chambre à soi : genres et corps en art »). Partis de journées d'études consacrées au 
corps dans les arts queer et féministes, le montage vidéo et le texte qui l'accompagnent inscrivent le travail et les rencontres effectuées à ce moment-là dans une archive académique féministe, et invitent à la poursuivre. Travail de traduction, ensuite : Sara Martinez a traduit pour GLAD! un entretien de Mariel Acosta avec Ernesto Cuba, entretien inédit en français, et dans lequel iels explorent «L'agitation au quotidien » à travers l'émergence d'une profusion de formes linguistiques « anarchistes ». Travail de diversification des temporalités, enfin, avec la troisième livraison de la chronique « Les genres récrits» (Daniel Elmiger), et la seconde des "Genres décrits» (Julie Abbou). Qu'il s'agisse des varia ou des dossiers thématiques, GLAD! protège ainsi précieusement sa tête des attaques des Lustucru en tout genre qui voudraient la lui reformater, et poursuit avec acharnement son travail d'édition féministe et politique!

$$
\begin{array}{r}
\text { Auréline Cardoso } \\
\text { et le collectif Arpège-EFiGiES Toulouse (Émilie } \\
\text { Blanc, Aude Ferrachat, Leslie Fonquerne, Bruna } \\
\text { Martins-Coelho, Héloïse Prévost, Agathe Roby- } \\
\text { Sapin, Marine Rouch, Marie Walin, Justine Zeller) } \\
\text { Charlotte Thevenet } \\
\text { et le comité de rédaction de la revue GLAD! (Julie } \\
\text { Abbou, Aron Arnold, Maria Candea, Alice Coutant, } \\
\text { Mona Gérardin-Laverge, Magali Guaresi, } \\
\text { Stavroula Katsiki, Noémie Marignier, Lucy } \\
\text { Michel, Émilie Née) }
\end{array}
$$

Nous remercions encore une fois les membres du comité de rédaction pour avoir ouvert ses pages à la publication des journées d'étude Arpège-EFiGiES Toulouse permettant ainsi la plus grande diffusion des savoirs coproduits par des universitaires et des militantes féministes. Nous espérons que ce dossier pourra donner quelques pistes d'action, à l'heure où les revendications féministes sont encore loin d'être satisfaites et où les régressions en termes d'acquis sociaux sont plus que probables.

Le collectif Arpège EFiGiES Toulouse

\section{BIBLIOGRAPHIE}

ANDRIAMANDROSo, Hanitra. 2017. " Déni de la "violence conjugale" et de la "violencia de género" : une clef de voûte des stratégies des groupes masculinistes en France et en Espagne. », Communication au colloque Antiféminismes et masculinismes d'hier et d'aujourd'hui, Université d'Angers, 4 mars 2017.

ARONSON, Pamela. 2015. « Féministes ou postféministes ?, Feminists or "Postfeminists" ? » Politix $109: 135-158$.

BARD, christine. 1999. Un siècle d'antiféminisme. Paris : Fayard. 
BARD, Christine. 2012. Le féminisme au-delà des idées reçues. Paris : Le Cavalier Bleu. BLAIS, Mélissa. 2012. «Y a-t-il un “cycle de la violence antiféministe” ? Les effets de l'antiféminisme selon les féministes québécoises » Cahiers du Genre 52(1) : 167-195.

BLAIS, Mélissa. 2017. «Penser les effets de l'antiféminisme comme facteur de transformation du mouvement féministe : le cas du Québec », Communication au colloque Antiféminismes et masculinismes d'hier et d'aujourd'hui, Université d'Angers, 4 mars 2017.

BLAIS, Mélissa \& DUPUIS-DÉRI, Francis. 2012. « Masculinism and the Antifeminist Countermovement » Social Movement Studies 11(1) : 21-39.

BLAIS, Mélissa \& DUPUIS-DÉRI, Francis. 2014. « Antiféminisme : pas d'exception française » Travail, genre et sociétés 32(2) : 151-156.

CASAS VILA, Gloria. 2009. « Médiation familiale : quelle place pour les violences conjugales?» Empan 2 (73) : 70-75

COSSY, valérie, MALBOIS, Fabienne, PARINI, Lorena \& LEMPEN, Silvia. 2009. « Édito : Imaginaires collectifs et reconfiguration du féminisme » Nouvelles Questions Féministes 28(1) : 4-13.

CÔTÉ, Isabelle \& LAPIERRE, simon. 2017. « La récupération de l'“aliénation parentale” au tribunal de la famille, par les antiféministes, au Québec », Communication au colloque Antiféminismes et masculinismes d'hier et d'aujourd'hui, Université d'Angers, 4 mars 2017.

DAUPHIN, sandrine. 2010. L'État et les droits des femmes. Rennes : Presses universitaires de Rennes. DeLPHY, Christine. 2001. L'ennemi principal. Penser le Genre. Paris : Éditions Syllepses.

DelPHY, Christine. 2010. «Retrouver l'élan du féminisme », in Un universalisme si particulier, DELPHY, Christine. Paris : Éditions Syllepses, 69-75.

DESCARRIES, Francine. 2005. « L'antiféminisme “ordinaire” » Recherches féministes 18(2) : 137-151. DEVREUX, Anne-Marie \& LAMOUReUX, Diane. 2012. « Les antiféminismes : une nébuleuse aux manifestations tangibles » Cahiers du Genre 52(1) : 7-22.

DUPUIS-DÉRI, Francis. 2013. « L'antiféminisme d'État » Lien social et Politiques 69 : 163-180.

DUPUIS-DÉRI, Francis. 2015. « Postféminisme et antiféminisme », in Les antiféminismes. Analyse d'un discours réactionnaire, DUPUIS-DERI, Francis. Les Éditions du Remue ménage : Montréal, 129-148.

FILLOD-CHABAUD, Aurélie. 2014. Au nom du père. Une sociologie comparative du militantisme paternel en France et au Québec. Thèse de doctorat, Florence, European University Institute.

GENZ, stéphanie. 2006. « Third way/ve: The politics of postfeminism » Feminist Theory 7(3): 333-353.

GOURARIER, Mélanie. 2017. Alpha mâle. Séduire les femmes pour s'apprécier entre hommes. Paris : Seuil. HALL, Elaine J. \& RODRIGUEZ, Marnie S. 2003. « The myth of postfeminism » Gender \& Society 17(6) : 878-902.

HAWKESWORTH, Mary. 2004. " The Semiotics of Premature Burial: Feminism in a Postfeminist Age » Signs 29(4): 961-985.

HIRSCHMAN, Albert. 1991. Deux siècles de rhétorique réactionnaire. Paris : Fayard.

KLAUS, Elisabeth. 2010. « Antiféminisme et féminisme élitiste en Allemagne : les termes du débat » Travail, genre et sociétés 24(2) : 151-165. 
LAMOUREUX, Diane. 2008. « Un terreau antiféministe », in Le mouvement masculiniste au Québec, l'antiféminisme démasqué, LAMOUREUx, Diane. Montréal : Les Éditions du Remue ménage, Montréal, $55-73$.

LEFORT, Étienne. 2017. «Qui parle, et de quoi, dans les groupes de paroles des associations pour le "droit des pères" ? ", Communication au colloque Antiféminismes et masculinismes d'hier et d'aujourd'hui, Université d'Angers, 4 mars 2017.

RABENORO, Mireille. 2012. « Le mythe des femmes au pouvoir, arme de l'antiféminisme à Madagascar » Cahiers du Genre 52(1) : 75-95.

\section{NOTES}

1. L'atelier Arpège-EFiGiES Toulouse est animé par de jeunes chercheuses en études genre issues de diverses disciplines (histoire, sociologie, anthropologie). Pour plus d'information sur l'atelier : https://efigies-ateliers.hypotheses.org/category/efigies-toulouse

2. Dans un article paru en avril dans Libération, Martine Storti dénonce cet antiféminisme qui se dit «féministe » venu de femmes publiant dans la revue d'extrême-droite Limite, et qui prônent un «féminisme intégral » contre la «médicalisation» du corps des femmes, par exemple. Il s'agit, en fait, d'un exemple typique d'écoblanchiment (greenwashing) des pires idées réactionnaires. "Attention, détournements de féminismes", Libération, 25 avril 20182018 http://www.liberation.fr/debats/2018/04/25/attention-detournements-de-feminismes_1645862, consulté le 9 juin 2018.

3. Pour des recherches déjà achevées voir par exemple Fillod-Chabaud (2014) ou Gourarier (2017), et pour des recherches en cours voir outre celles présentées dans ce dossier Andriamandroso (2017) ou Lefort (2017).

4. Voir l'exposition virtuelle de Christine Bard et Valérie Neveu «Visages du suffragisme français ", en particulier la section « Les évolutions de l'argumentaire antiféministe », sur le site Muséa http://musea.univ-angers.fr, consulté le 09 juin 2018

5. Voir l'exposition de Bard et Neveu citée précédemment.

6. Nous faisons référence aux prises de parole publiques d'actrices qui ont dénoncé au cours de l'été 2017 les violences sexuelles exercées par le producteur américain Harvey Weinstein.

7. Notamment Lola Lafon dans l'émission « Harcèlement sexuel : le grand déballage ", Le nouveau rendez-vous, France Inter, 23 octobre 2017. https://www.franceinter.fr/emissions/le-nouveaurendez-vous/le-nouveau-rendez-vous-23-octobre-2017-0

8. "Contre la pénalisation du harcèlement de rue ", Libération, 26 septembre 2017. http:// www.liberation.fr/debats/2017/09/26/contre-la-penalisation-du-harcelement-de-rue_1599121

\section{INDEX}

Thèmes : Recherches

Mots-clés : argumentation, misogynie, masculinisme, rhétorique, antiféminisme

Keywords : argumentation, misogyny, masculinism, rhetoric, antifeminism 


\section{AUTEURS}

\section{AURÉLINE CARDOSO}

Auréline Cardoso est doctorante en sociologie à l'Université Toulouse II Jean Jaurès, au CERTOP. Sa thèse porte sur l'usure au travail dans les associations féministes. Elle est co-fondatrice du collectif Arpège-EFiGiES-Toulouse, créé en septembre 2015. Ce collectif vise à proposer des espaces d'échange de savoirs autour du genre en tentant de placer savoirs professionnels, militants, pratiques et académiques sur un pied d'égalité.

\section{CHARLOTTE THEVENET}

Doctorante en littérature française à University College London (SELCS, French), Charlotte Thevenet prépare une thèse sur la rhétorique du commentaire chez Jacques Derrida. 